\title{
ÉDITORIAL
}

\section{Le CMAJ et la lutte contre le racisme}

\author{
Kirsten Patrick MBBCh MSc
}

Citation : CMAJ 2021 March 29;193:E453-4. doi : 10.1503/cmaj.210494-f

Voir la version anglaise de l'article ici : www.cmaj.ca/lookup/doi/10.1503/cmaj.210494

A u début de l'année 2021, le CMAJ a publié des articles appelant à mettre fin au racisme dans le domaine de la santé et le milieu universitaire médical au Canada ${ }^{1-3}$. Nous avons reçu des commentaires encourageants sur ces articles. J'ai toutefois été troublée par certains courriels et messages vocaux de lecteurs qui exprimaient leur indignation à l'égard du fait que le CMAJ offre une tribune à l'idée selon laquelle le racisme existe, que ce soit au Canada ou dans la profession médicale. Une personne a affirmé sans détour qu'«il n'y a pas de racisme en médecine ». Une autre, tout en admettant que des actes racistes se produisent à l'occasion au niveau interpersonnel, a déclaré que le racisme structurel n'existait pas au Canada.

Je vous écris aujourd'hui pour affirmer de façon catégorique la position du CMAJ à ce sujet : le racisme systémique est présent dans la société canadienne et dans ses systèmes de soins de santé. Mon but ici n'est pas de débattre de l'existence du racisme au Canada. De nombreuses preuves et de nombreux témoignages attestent de son existence. J'invite les lecteurs à en prendre connaissance. Comme le CMAJ fait partie du système qui produit un savoir médical, il fait et a fait partie du problème. Il est essentiel que nous reconnaissions notre part de responsabilité et que nous prenions des mesures pour faire partie de la solution.

Les revues médicales ont du pouvoir. Ces revues, et ceux qui travaillent à leur publication, figurent parmi les gardiens de l'information qui guident l'élaboration des politiques et la prestation des services dans les systèmes de santé. Les rédacteurs en chef disposent de tribunes puissantes qu'ils peuvent utiliser pour influencer la recherche scientifique et les discours politiques. Par conséquent, la diversité des personnes qui participent à la prise de décisions éditoriales doit refléter la diversité des intervenants en santé. Les voix marginalisées doivent être entendues pour qu'un changement s'opère.

Les revues scientifiques du monde entier ont réagi aux événements de 2020 en publiant des éditoriaux, des articles et des commentaires sur les conséquences du racisme, exprimant avec vigueur leur solidarité avec le mouvement antiraciste. Certaines ont publié des éditions spéciales sur le racisme en médecine. Pourtant, des critiques ont mis en doute leurs motivations; notamment, un chercheur universitaire a fait remarquer que " certaines revues universitaires qui ont récemment prétendu s'engager en faveur de l'équité raciale publient des travaux critiques uniquement sous la forme de commentaires et d'articles d'opinion, alors que les travaux critiques empiriques fondés sur la théorie ne résistent pas au processus d'examen par les pairs ${ }^{4} »$.

Il est important d'entendre ces critiques et d'y réfléchir plutôt que d'être sur la défensive. Ces dernières années, le CMAJ a publié un bon volume de recherches mettant en évidence des disparités en santé entre les groupes racisés et les personnes blanches au Canada. Cela dit, se contenter de montrer que les personnes noires ou les Autochtones au Canada présentent des issues comparativement plus défavorables dans certains domaines de la santé, ce n'est pas la même chose que démontrer que le racisme est à l'origine de ces inégalités en santé; c'est aussi très loin de s'attaquer aux problèmes structurels sous-jacents.

Les chercheurs universitaires du domaine des sciences sociales ont produit des preuves des effets des politiques racistes depuis plus d'un siècle ${ }^{5,6}$. Au cours des dernières décennies, la recherche médicale a intégré l'interrelation entre l'expérience sociale et les résultats en santé, et la mesure dans laquelle certains déterminants sociaux affectent l'état de santé est désormais bien comprise. Cependant, il est plus facile de mesurer le revenu, la situation de logement et l'éducation, et d'établir des corrélations avec la santé, que de quantifier les effets cumulatifs d'une vie entière d'exclusion sociale et d'oppression. De ce fait, notre compréhension des effets du racisme sur la santé (plutôt que des corrélations avec la race ou l'ethnicité) est moins approfondie. Le manque de données détaillées est d'ailleurs l'un des obstacles qui empêchent de répondre aux questions pertinentes sur les effets du racisme sur la santé. En termes clairs, bien que nous demandions aux gens de s'identifier comme faisant partie d'un groupe racisé, nous leur demandons rarement de fournir des données sur leur expérience du racisme.

Le CMAJ ne peut pas influencer directement la nature de la recherche financée et menée au Canada, mais il peut offrir une 
tribune aux personnes qui souhaitent s'exprimer sur les recherches à mener, les données à recueillir et les méthodes à utiliser pour permettre une étude adéquate du racisme dans le domaine de la santé. Nous attendons avec impatience de recevoir des propositions de recherche innovantes sur des interventions visant à réduire à la fois le racisme dans le domaine de la santé et les effets du racisme sur la santé.

En tant que rédactrice en chef par intérim du CMAJ, je m'efforcerai de promouvoir un programme antiraciste. À court terme, pour modifier l'équilibre du pouvoir parmi les personnes qui prennent les décisions éditoriales, je tenterai d'ajouter à l'équipe éditoriale du CMAJ un rédacteur spécialisé dans la théorie critique de la race, et d'ajouter au comité consultatif éditorial actuel de la revue un membre capable de donner des conseils avisés sur l'élaboration de politiques antiracistes. L'équipe éditoriale actuelle du CMAJ recevra une formation sur les préjugés inconscients et de la formation continue sur les nouveaux développements pertinents concernant les méthodes de recherche. Nous continuerons à commander et à publier des articles qui traitent de façon critique des conséquences du racisme sur la santé des personnes au Canada et qui cherchent à présenter de véritables solutions.

Pour certains lecteurs, cet éditorial est attendu depuis longtemps. Je remercie les personnes qui ont déjà signalé le racisme structurel au CMAJ et qui ont patiemment pris le temps de m'éduquer sur le sujet. J'attends des lecteurs du CMAJ qu'ils me tiennent responsable de ces intentions déclarées.

\section{Références}

1. Dryden $\mathrm{O}$, Nnorom $\mathrm{O}$. Time to dismantle systemic anti-Black racism in medicine in Canada. CMAJ 2021;193:E55-7.

2. Datta G, Siddiqi A, Lofters A. Transforming race-based health research in Canada. CMAJ 2021;193:E99-100.

3. Sharda S. Dhara, Alam F. Not neutral: reimagining antiracism as a professional competence. CMAJ 2021;193:E101-2.

4. @Arrianna_Planey Planey AM. “I'm observing that some academic journals that have recently claimed to have a commitment to racial equity are only publishing critical work in the form of commentaries \& op-eds, while theoretically-grounded, empirical critical work does not get past their peer review process." Twitter 2021 Feb. 9. Accessible ici : https://twitter.com/Arrianna_Planey/ status/1359273820947939332 (consulté le 9 fév. 2021).

5. DuBois WEB. The Philadelphia Negro: a social study. Philadelphia: University of Pennsylvania Press; 1996. Accessible ici : www.jstor.org/stable/j.ctt3fhpfb (consulté le 6 mars 2021).

6. Jones CP, Truman BI, Elam-Evans LD, et al. Using "socially assigned race" to probe white advantages in health status. Ethn Dis 2008;18:496-504.

Intérêts concurrents : Voir www.cmaj.ca/site/misc/cmaj_staff.xhtml.

Affiliations : Rédactrice en chef par intérim, CMAJ.

Propriété intellectuelle du contenu : Il s'agit d'un article en libre accès distribué conformément aux modalités de la licence Creative Commons Attribution (CC BY-NC-ND 4.0), qui permet l'utilisation, la diffusion et la reproduction dans tout média à la condition que la publication originale soit adéquatement citée, que l'utilisation se fasse à des fins non commerciales (c.-à-d., recherche ou éducation) et qu'aucune modification ni adaptation n'y soit apportée. Voir : https://creativecommons.org/licenses/by-nc-nd/4.0/deed.fr.

Correspondance : Rédaction du CMAJ, editorial@cmaj.ca 